\section{OPEN ACCESS}

Edited by:

Shuai Le,

Army Medical University, China

Reviewed by:

Hang Yang,

Wuhan Institute of Virology, Chinese Academy of Sciences (CAS), China

Tang Fang,

Nanjing Agricultural University, China

${ }^{*}$ Correspondence:

Jingmin Gu

jingmin0629@163.com

${ }^{t}$ These authors have contributed equally to this work

Specialty section:

This article was submitted to

Virology,

a section of the journal

Frontiers in Microbiology

Received: 28 February 2021 Accepted: 30 March 2021

Published: 22 April 2021

Citation:

Wang Z, Cai R, Wang G, Guo Z, Liu X, Guan Y, Ji Y, Zhang H, Xi $H$, Zhao $R$, Bi L, Liu S, Yang L, Feng $X$ Sun C, Lei L, Han W and Gu J (2021)

Combination Therapy of Phage

vB_KpnM_P-KP2 and Gentamicin Combats Acute Pneumonia Caused by K47 Serotype Klebsiella pneumoniae.

Front. Microbiol. 12:674068. doi: 10.3389/fmicb.2021.674068

\title{
Combination Therapy of Phage
} vB_KpnM_P-KP2 and Gentamicin Combats Acute Pneumonia Caused by K47 Serotype Klebsiella pneumoniae

Zijing Wang ${ }^{1+}$, Ruopeng Cai2t, Gang Wang ${ }^{1+}$, Zhimin Guo ${ }^{3 \dagger}$, Xiao Liu', Yuan Guan', Yalu Ji', Hao Zhang ${ }^{1}$, Hengyu Xi', Rihong Zhao ${ }^{1}$, Lanting Bi ${ }^{1}$, Shanshan Liu ${ }^{4}$, Li Yang ${ }^{1}$, Xin Feng ${ }^{1}$, Changjiang Sun ${ }^{1}$, Liancheng Lei ${ }^{1}$, Wenyu Han ${ }^{1,5}$ and Jingmin Gu ${ }^{1,5 *}$

${ }^{1}$ Key Laboratory of Zoonosis Research, Ministry of Education, College of Veterinary Medicine, Jilin University, Changchun, China, ${ }^{2}$ College of Animal Science and Technology, Jilin Agricultural University, Changchun, China, ${ }^{3}$ Department of Clinical Laboratory, The First Hospital of Jilin University, Changchun, China, ${ }^{4}$ Department of Chinese Journal of Veterinary Science, Jilin University, Changchun, China, ${ }^{5}$ Jiangsu Co-Innovation Center for the Prevention and Control of Important Animal Infectious Disease and Zoonose, Yangzhou University, Yangzhou, China

Klebsiella pneumoniae ( $K$. pneumoniae) is an important nosocomial and community acquired opportunistic pathogen which causes various infections. The emergence of multi-drug resistant (MDR) K. pneumoniae and carbapenem-resistant hypervirulent K. pneumoniae (CR-hvKP) has brought more severe challenge to the treatment of $K$. pneumoniae infection. In this study, a novel bacteriophage that specifically infects K. pneumoniae was isolated and named as vB_KpnM_P-KP2 (abbreviated as P-KP2). The biological characteristics of P-KP2 and the bioinformatics of its genome were analyzed, and then the therapeutic effect of P-KP2 was tested by animal experiments. $\mathrm{P}-\mathrm{KP} 2$ presents high lysis efficiency in vitro. The genome of P-KP2 shows homology with nine phages which belong to "KP15 virus" family and its genome comprises 172,138 bp and 264 ORFs. Besides, P-KP2 was comparable to gentamicin in the treatment of lethal pneumonia caused by K. pneumoniae W-KP2 (K47 serotype). Furthermore, the combined treatment of P-KP2 and gentamicin completely rescued the infected mice. Therefore, this study not only introduces a new member to the phage therapeutic library, but also serves as a reference for other phage-antibiotic combinations to combat MDR pathogens.

Keywords: bacteriophage, Klebsiella pneumoniae, genome sequencing, bioinformatics analysis, phage therapy

\section{INTRODUCTION}

As the second-ranked nosocomial infection-causing-pathogens, Klebsiella pneumoniae (K. pneumoniae) causes fatal systemic infections (Paczosa and Mecsas, 2016). The presence of at least 79 serotypes greatly increases the complexity of treatment for these bacterial infections (Hsu et al., 2013; Pan et al., 2015). What's more, the existing antibiotics have failed to cure clinical infections caused by multidrug-resistant (MDR) K. pneumoniae, such as 
extended-spectrum $\beta$-lactamase $\quad$ (ESBL)-producing and carbapenemase [such as $K$. pneumoniae carbapenemases (KPC), metallo $\beta$-lactamases (MBL), and oxacillinase-48-type carbapenemases (OXA-48)]-producing strains (Navon-Venezia et al., 2017). In addition, the recent emergence of carbapenemresistant hypervirulent Klebsiella pneumoniae (CR-hvKP) has further exacerbated the dilemma of antibiotic treatment, provoking the need for alternative therapies (Gu et al., 2018).

Bacteriophages (phages) are bacterial viruses that specifically recognize, infect, and replicate inside a host bacterium. It has been reported that there are more than $10^{31}$ phage particles in the biosphere. This massive phage diversity has a marked effect on the environment, ecology, and bacterial evolution (Davies et al., 2016). Owing to their specific bactericidal abilities, phages have been considered as therapeutic agents since the early 1920s. However, the development of this therapy has been hampered by the widespread use of antibiotics (Nobrega et al., 2015). Recently, due to the global emergence of multidrug-resistant bacteria, phage therapy has been experiencing a renaissance for its ability to combat their antibiotic-resistant host specifically. Until now, phage therapy have already been developed in various bacterial species including MDR K. pneumoniae and have made some achievements. Studies based on mice as animal models have shown that phages have good therapeutic effects on pneumonia (Anand et al., 2020), liver abscess (Lin et al., 2014), burn infection (Chadha et al., 2017), and bacteremia (Kaabi and Musafer, 2019) caused by K. pneumoniae. Not only that, phage therapy has also been applied in clinical practice. Medical workers have confirmed that multiple rounds of phage administration have a significant curative effect on refractory urinary tract infections triggered by MDR K. pneumoniae (Bao et al., 2020). Although phage therapy has great potential application prospects, both the phage resistance of bacteria and phage elimination by the immune system are the main challenges (Hodyra-Stefaniak et al., 2015; Hampton et al., 2020). However, the combination therapy of phage and antibiotics can effectively increase the sensitivity of target strains to antibiotics and reduce the probability of phage resistance mutations, thereby providing a development direction for controlling bacterial infections caused by MDR strains (Abedon, 2019).

In the present work, a novel myophage that specifically infects K. pneumoniae was isolated and named as vB_KpnM_P-KP2 (abbreviated as P-KP2). The biological characteristics of this phage such as morphology and one-step growth curve were measured. Besides, the genetic background of P-KP2 was revealed by bioinformatics analysis, and the powerful therapeutic effect of the phage combined with gentamicin on lung infection caused by K. pneumoniae W-KP2 (K47 serotype MDR K. pneumoniae strain) was also confirmed.

\section{MATERIALS AND METHODS}

\section{Animals}

Female C57BL/6J mice (18-20 g) were purchased from Liaoning Changsheng Biotechnology Co., Ltd. (Benxi, Liaoning, China). All animal experiments were performed in strict accordance with the Regulations for the Administration of Affairs Concerning Experimental Animals approved by the State Council of the People's Republic of China and the Animal Welfare and Research Ethics Committee at University.

\section{Bacterial Strains and Culture Conditions}

K. pneumoniae W-KP2 was isolated from sputum provided by Zhimin Guo and confirmed to be K. pneumoniae using 16S rRNA sequence analysis after PCR amplification with the universal primers (27F: AGAGTTTGATCCTGGCTCAG and 1429R: GGTTACCTTGTTACGACTT) (Sunagawa et al., 2009). The serotype of $K$. pneumoniae was identified by the universal primer (F: GGGTTTTTATCGGGTTGTAC and R: $5^{\prime}-3^{\prime}$ TTCAGCTGGATTTGGTGG) according to the previous description (Pan et al., 2013). The minimum inhibitory concentration (MIC) of antibiotics listed in Supplementary Table 1 for K. pneumoniae W-KP2 (0.5-0.63 MacFarland) was assessed using the VITEK ${ }^{\circledR} 2$ Compact system with VITEK ${ }^{\circledR} 2$ AST-GN 09 Card (bioMérieux, Marcy-l'Étoile, France) according to the manufacturer's instructions. The strain was routinely inoculated in lysogeny broth (LB) broth and then propagated on an orbital shaker (180 $\mathrm{rpm} / \mathrm{min})$.

\section{Phage Isolation, Purification, and Host Spectrum Determination}

Phage P-KP2 was isolated by using $K$. pneumoniae W-KP2 as the host strain from sewage samples collected from the Changchun sewer system $\left(43^{\circ} 92^{\prime} \mathrm{N}, 125^{\circ} 25^{\prime} \mathrm{E}\right)$ (Changchun, Jilin Province, China) according to the previously described method (Ji et al., 2019). In brief, W-KP2 was incubated with sewage samples in $\mathrm{LB}$ broth at $37^{\circ} \mathrm{C}$ overnight. After $15 \mathrm{~min}$ of centrifugation $\left(4^{\circ} \mathrm{C}, 10,000 \times \mathrm{g}\right)$ to remove the precipitate, the supernatant was filtered by $0.22-\mu \mathrm{m}$ filters (Millipore, Billerica, MA, United States). Phage P-KP2 was purified by the doublelayer agar plate method and then stored at $4^{\circ} \mathrm{C}$ or $-80^{\circ} \mathrm{C}$ in glycerol $(3: 1[v / v])$. To determine its host spectrum, $5 \mu \mathrm{L}$ of phage P-KP2 suspension was spot tested against 80 clinical $K$. pneumoniae strains preserved in the laboratory, by the doublelayer agar plate method (Cai et al., 2019b).

\section{Growth Characteristics of the Phage}

To determine the titers of phage P-KP2 corresponding to different multiplicity of infection (MOI), the phage was added to fresh $K$. pneumoniae $\mathrm{W}-\mathrm{KP} 2$ culture with final concentration of $2.0 \times 10^{7} \mathrm{CFU} / \mathrm{mL}\left(\mathrm{OD}_{600} \approx 0.4\right)$ at different MOIs (phage $/$ bacteria $=0.00000001,0.0000001,0.000001,0.00001$, $0.0001,0.001,0.01,0.1,1,10$, and 100). After the mixed culture was incubated for $5 \mathrm{~h}$ at $37^{\circ} \mathrm{C}(180 \mathrm{rpm})$, the phage titers were measured by the double-layer agar plate method after serial dilution (Gong et al., 2016).

To determine the one-step growth curve of $\mathrm{P}-\mathrm{KP} 2$, the phage was added to $\mathrm{W}$-KP2 culture $\left(5.0 \times 10^{5} \mathrm{CFU} / \mathrm{mL}\right)$ at an MOI of 0.1 . After $10 \mathrm{~min}$ of incubation at $37^{\circ} \mathrm{C}$, the mixture was centrifuged at $12,000 \times \mathrm{g}$ for $5 \mathrm{~min}$ at $4^{\circ} \mathrm{C}$. The pellet was resuspended in $10 \mathrm{~mL}$ of fresh $\mathrm{LB}$ broth followed by incubation at $37^{\circ} \mathrm{C}$ with shaking at $180 \mathrm{rpm}$. Cultures were collected 
every $10 \mathrm{~min}$ for $180 \mathrm{~min}$ and were filtered by $0.22-\mu \mathrm{m}$ filters (Millipore, Billerica, MA, United States). Finally, plaque assays were used to quantify the lysate titers at different time intervals (Gadagkar and Gopinathan, 1980).

\section{Concentration and Purification of the Phage}

Phage P-KP2 was concentrated and purified according to the previous description for morphological observation and genome extraction (Sambrook and Russell, 2006). Briefly, $100 \mu \mathrm{L}$ of phage $\mathrm{P}-\mathrm{KP} 2$ suspension $\left(2.0 \times 10^{7} \mathrm{PFU} / \mathrm{mL}\right)$ was added to $1 \mathrm{~L}$ of K. pneumoniae $\mathrm{W}-\mathrm{KP} 2\left(\mathrm{OD}_{600} \approx 0.4\right)$. After shaking culture $(180 \mathrm{rpm})$ for $4 \mathrm{~h}$, the lysates were centrifuged for $20 \mathrm{~min}\left(4^{\circ} \mathrm{C}\right.$, $4,000 \times \mathrm{g})$ to recover the supernatant, and DNase I and RNase A $(1 \mu \mathrm{g} / \mathrm{mL})$ were added. Followed by incubation at $25^{\circ} \mathrm{C}$ for $30 \mathrm{~min}, 1 \mathrm{M} \mathrm{NaCl}$ was then added to the supernatants (ice bath for $1 \mathrm{~h}$ ). After the addition of polyethylene glycol 8000 (PEG8000) $(10 \%[w / v])$, the mixture was incubated in an ice bath overnight. Next, the phage particles were collected by centrifugation for $10 \mathrm{~min}\left(4^{\circ} \mathrm{C}, 10,000 \times \mathrm{g}\right)$ and resuspended in $2 \mathrm{~mL} \mathrm{SM}$ buffer. The concentrated phage was purified by cesium chloride $(\mathrm{CsCl})$ density gradient centrifugation $\left(4^{\circ} \mathrm{C}, 35,000 \times \mathrm{g}, 3 \mathrm{~h}, \mathrm{CsCl}\right.$ gradient: $1.32,1.45,1.50$, and $1.70 \mathrm{~g} / \mathrm{mL})$, and then the light blue phage band was carefully collected and subjected to dialysis before being stored at $4^{\circ} \mathrm{C}$.

\section{Morphological Observation of the Phage}

After negative staining with phosphotungstic acid (PTA) $(2 \%[w / v])$, the morphology of the concentrated phage $\left(2.5 \times 10^{10} \mathrm{PFU} / \mathrm{mL}\right)$ was examined using a transmission electron microscope (TEM) (JEOL JEM-1200EXII, Japan Electronics and Optics Laboratory, Tokyo, Japan) at an acceleration voltage of $80 \mathrm{kV}$.

\section{Sequencing and Bioinformatics Analysis of the Phage Genome}

The genome was extracted from the concentrated and purified phage preparations using a viral genome extraction kit (Omega Bio-Tek Inc., Doraville, GA, United States) [Quality criteria for DNA samples: $\mathrm{OD}_{260 / 280}=1.8-2.0, \mathrm{OD}_{260 / 230}=2.0-$ 2.2, $\mathrm{RIN}=6.0-8.0, \geq 10 \mu \mathrm{g}$ in total $(\geq 100 \mathrm{ng} / \mu \mathrm{L})]$. Wholegenome sequencing was performed by using Illumina HiSeq 2500 sequencing. The genome sequences were assembled using Roche Newbler v.2.8 (Margulies et al., 2005). Potential open reading frames (ORFs) were identified using GeneMarkS (Georgia Institute of Technology, Atlanta, GA, United States) [parameter settings: (sequence type: phage; output format for gene prediction: LST; output options: gene nucleotide sequence)] (Besemer et al., 2001). The gene alignment and ORF annotation were performed using BLASTN (nucleotide collection database, Megablast) and BLASTP [non-redundant protein sequence database, PSI-BLAST (Threshold $=0.005)$ ], respectively, from the National Center for Biotechnology Information (NCBI) (Altschul et al., 1997). The schematic of the phage genome with predicted ORFs was generated using CLC Main Workbench, version 8.0.1 (CLC Bio-Qiagen, Aarhus, Denmark). The circular view of the phage genome was illustrated using CGView ${ }^{1}$ (Stothard and Wishart, 2005) and termini of the phage was identified by PhageTerm ${ }^{2}$ (Garneau et al., 2017). Genome comparisons among P-KP2 and homologous phages were performed by Mauve 2.3.1 (Darling et al., 2004). After genes of terminase large subunit, major capsid protein and DNA polymerase were aligned by ClustalW, phylogenetic trees of phages were constructed using Neighbor-Joining Method (100 bootstrap replicates) by PHYLIP (version 3.697) (Shimada and Nishida, 2017). Additionally, domain analyses were performed by HHpred at MPI bioinformatics Toolkit ${ }^{3}$ (Zimmermann et al., 2018).

\section{Therapeutic Effects of P-KP2 and Gentamicin Against $K$. pneumoniae W-KP2}

After $K$. pneumoniae W-KP2 was cultured to exponential growth phase $\left(2.0 \times 10^{9} \mathrm{CFU} / \mathrm{mL}\right)$, the bacterial solution was concentrated by centrifugation and suspended in phosphate buffered saline (PBS) (adjust to $5.0 \times 10^{10} \mathrm{CFU} / \mathrm{mL}$ ). To determine the virulence of $\mathrm{W}-\mathrm{KP} 2$, mice were anesthetized intraperitoneally with ketamine $(100 \mathrm{mg} / \mathrm{kg})$ and xylazine $(10 \mathrm{mg} / \mathrm{kg}$ ) followed by intranasal inoculation with $50 \mu \mathrm{L}$ of W-KP2 suspension of different dilutions $\left(5.0 \times 10^{5}, 5.0 \times 10^{6}\right.$, $5.0 \times 10^{7}, 5.0 \times 10^{8}, 1.0 \times 10^{9}$, or $2.5 \times 10^{9} \mathrm{CFU} /$ mouse, $n=10$ per group), and then the minimum bacterial dose that triggered $100 \%$ death within 7 -days [minimum lethal dose (MLD)] was determined (Xia et al., 2016). Finally, all surviving mice were euthanized by intravenous injection of Fatal Plus (sodium pentobarbital) $(100 \mathrm{mg} / \mathrm{kg})$.

The therapeutic effects of phage P-KP2, gentamicin and phage-antibiotic combination in the treatment of acute pneumonia caused by K. pneumoniae W-KP2 were evaluated based on the mouse model. Mice were challenged intranasally with $2 \times \operatorname{MLD}\left(1.0 \times 10^{9} \mathrm{CFU} /\right.$ mouse $)$ of $\mathrm{W}-\mathrm{KP} 2$ in reference to our previous study (Cheng et al., 2017). At $1 \mathrm{~h}$ post infection, mice were treated intranasally with $\mathrm{P}-\mathrm{KP} 2$ after concentration $\left(1.0 \times 10^{7} \mathrm{PFU} / \mathrm{mouse}, 1.0 \times 10^{8} \mathrm{PFU} / \mathrm{mouse}\right.$ or $1.0 \times 10^{9}$ $\mathrm{PFU} / \mathrm{mouse})$, gentamicin $(1.5 \mathrm{mg} / \mathrm{kg})$, or P-KP2 $\left(1.0 \times 10^{9}\right.$ $\mathrm{PFU} / \mathrm{mouse}$ ) in combination with gentamicin (gentamicin was administered intranasally at $30 \mathrm{~min}$ after P-KP2 administration), respectively. The untreated group was administered with PBS buffer after W-KP2 challenge. The survival rate, body weight and health status in each group were recorded within 7 days follow-up period.

Three mice selected randomly from each treatment group ( $n=30$ per group) at every time point were euthanized using Fatal Plus (sodium pentobarbital) $(100 \mathrm{mg} / \mathrm{kg}$ ) every $24 \mathrm{~h}$ (lasting for 7 days) after infection. Blood was collected from the tail vein of these mice and then the bacterial load was determined by plating after serial dilution. Their lungs were carefully removed and photographed. After fixation with $4 \%$ formalin, the left lungs were embedded with paraffin and stained with hematoxylin and eosin (H\&E) (Feldman and Wolfe, 2014), followed by

\footnotetext{
${ }^{1}$ http://wishart.biology.ualberta.ca/cgview/

${ }^{2}$ https://sourceforge.net/projects/phageterm

${ }^{3}$ https://toolkit.tuebingen.mpg.de/\#/tools/hhpred
} 
histopathological analysis through a microscope (Olympus CX41; Olympus America, Center Valley, PA, United States). After weighing, the right lung tissues were immediately lysed and homogenized, then the lung homogenates were serially diluted and bacterial loads were determined by plating. Simultaneously, the phage titers in lung tissues were detected using the doublelayer agar plate method. For enzyme-linked immunosorbent assay, the supernatants of lung homogenates were obtained according to our previous method $\left(4^{\circ} \mathrm{C}, 5,000 \times \mathrm{g}, 10 \mathrm{~min}\right)$ (Cai et al., 2019a). The concentrations of interleukin $1 \beta$ (IL$1 \beta$ ), interleukin 6 (IL-6), tumor necrosis factor- $\alpha$ (TNF- $\alpha$ ), and interferon- $\gamma($ IFN- $\gamma)$ in the supernatants were measured by ELISA kits (eBioscience, San Diego, CA, United States) according to the manufacturer's instructions.

\section{Statistical Analysis}

Survival curve analyses were performed using log-rank (MantelCox) test. While other statistical data presented in this study were processed by One-way analysis of variance (ANOVA). GraphPad Prism 6 (GraphPad Software, Inc., San Diego, CA, United States) was utilized for chart generation. $P<0.05$ were considered statistically significant. Error bars represented standard error of the mean.

\section{RESULTS}

\section{The Characteristics of $K$. pneumoniae W-KP2 and Phage P-KP2}

Since sequencing result of $\mathrm{W}-\mathrm{KP} 2 w z c$ gene was consistent with K47 serotype reference sequences, $K$. pneumoniae $\mathrm{W}-\mathrm{KP} 2$ was identified as belonging to the K47 serotype (Supplementary Figure 1), which was resistant to the antibiotics listed in Supplementary Table 1, except for gentamicin, indicating that it is a MDR strain that may lead to ineffective antibiotic treatment.

A Klebsiella phage named vB_KpnM_P-KP2 (abbreviated as $\mathrm{P}-\mathrm{KP} 2$ ) was isolated using $K$. pneumoniae $\mathrm{W}-\mathrm{KP} 2$ in this study, and the initial titer of phage P-KP2 obtained after phage enrichment was $2.0 \times 10^{7} \mathrm{PFU} / \mathrm{mL}$. The phage formed small circular translucent plaques (diameter $<1 \mathrm{~mm}$ ) on the lawns of $K$. pneumoniae W-KP2 (Figure 1A). P-KP2 showed a prolate head with icosahedral structure (105 $\pm 5 \mathrm{~nm}$ in length and $79 \pm 3 \mathrm{~nm}$ in diameter $)$ and a long contractile tail $(100 \pm 10 \mathrm{~nm})$ with a baseplate as observed with TEM (Figure 1B), which indicated that it belongs to the family Myoviridae. Among the $80 \mathrm{~K}$. pneumoniae strains tested, phage P-KP2 could form both spots and plaques on 10 of them, including W-KP2 (Supplementary Table 2).

Phage titer of P-KP2 after propagation was highest at MOI 0.000001 with a titer of $7.5 \times 10^{7} \mathrm{PFU} / \mathrm{mL}$ (Figure $2 \mathrm{~A}$ ). Besides, the latent period of this phage was $30 \mathrm{~min}$, after which the number of viral particles was rapidly increased. The proliferation of P-KP2 took about another $30 \mathrm{~min}$ to reach the growth plateau phase with a burst size of $204 \mathrm{PFU} /$ cell (Figure 2B).

\section{Bioinformatics Analyses of P-KP2 Genome}

The genome of phage P-KP2 is a linear double-stranded DNA that is comprised of 172,138 bp with a $\mathrm{G}+\mathrm{C}$ content of $41.9 \%$, and the termini of the phage genome is located at 38,590 bp (Supplementary Figure 2). Comparative analysis of the complete nucleotide sequence indicates that several phages

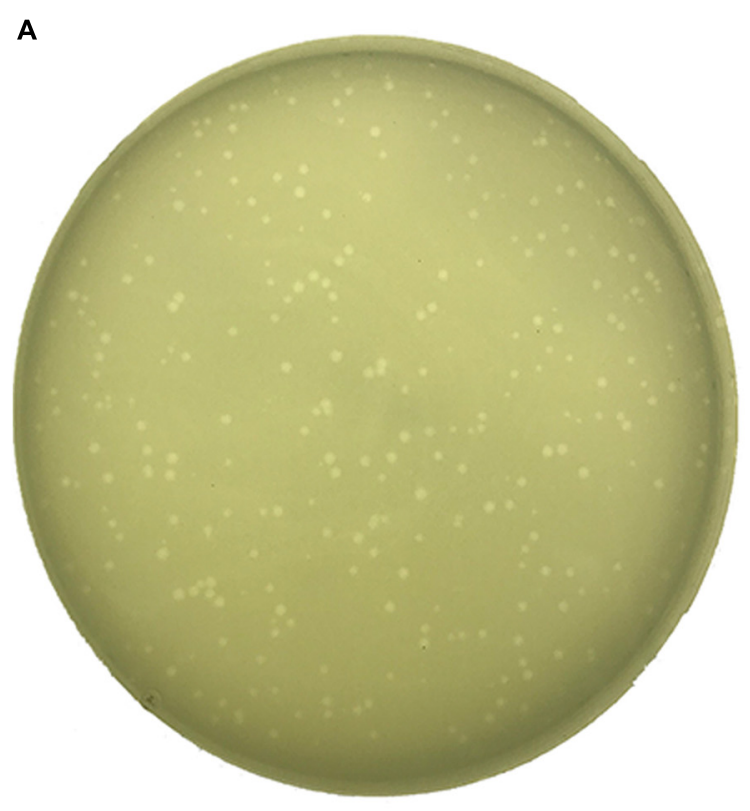

$\mathbf{B}$

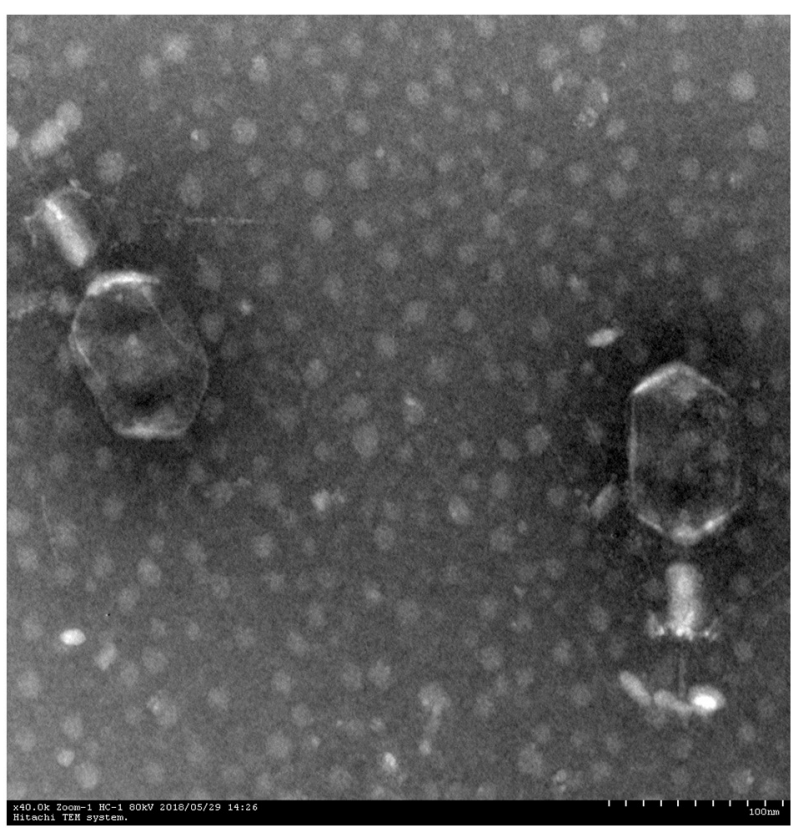

FIGURE 1 | The morphology of phage P-KP2. (A) The plaques formed by phage P-KP2 on the lawns of K. pneumoniae W-KP2. (B) The morphology of phage P-KP2 was determined by transmission electron microscopy (TEM). The scale bars represent $100 \mathrm{~nm}$. 

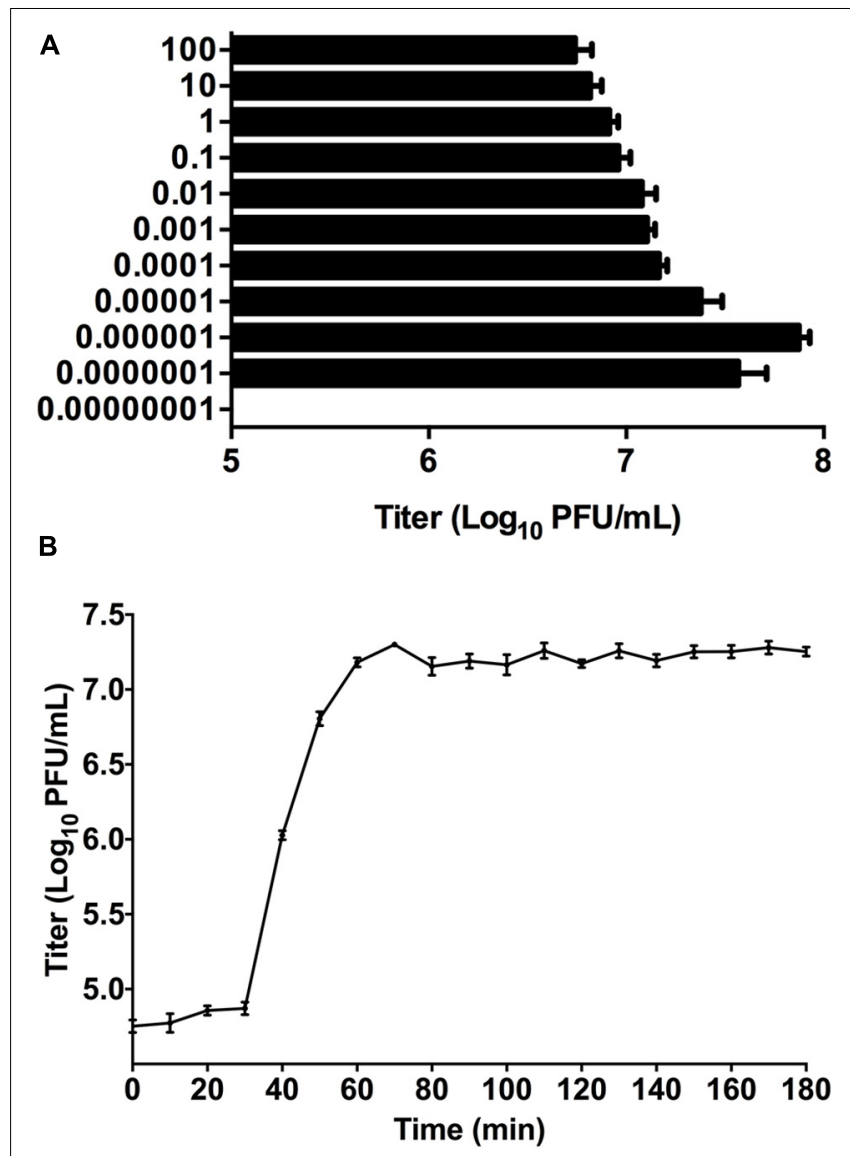

FIGURE 2 | The growth characteristics of P-KP2. (A) Titers of the phage under different $\mathrm{MOI}$ (phage/bacteria $=0.00000001,0.0000001,0.000001$, $0.00001,0.0001,0.001,0.01,0.1,1,10$, and 100), as indicated in the Y-axis. At the $\mathrm{MOI}$ of 0.000001 , P-KP2 reached maximum titers. (B) One-step growth curve of $\mathrm{P}-\mathrm{KP} 2$ was carried out at $\mathrm{MOI}=0.1$. Each data is shown as means \pm SEM from three biological experiments.

show high similarity to P-KP2, including Enterobacter phage phiEap-3 (Zhao et al., 2019), Klebsiella phage Matisse (Provasek et al., 2015), Klebsiella phage KP27, Klebsiella phage KP15, Klebsiella phage PMBT1 (Koberg et al., 2017), Klebsiella phage KOX10, Klebsiella phage Miro (Mijalis et al., 2015), Escherichia phage phT4A (Pereira et al., 2017), and Klebsiella phage KOX8 (Table 1), which can be classified as a member of pseudo-Teven myophages which belong to "KP15likevirus" genus. Multiple genome alignment confirmed that these phages have parallel gene functional modules (Figure 3). Phylogenetic analyses of the large terminase subunits, major capsid proteins and DNA polymerases showed that P-KP2 has close evolutionary relationships with Klebsiella phage KP27 (Figure 4).

The complete genome of P-KP2 includes 264 predicted ORFs. Among all the ORFs, 120 are transcribed in one orientation, and 144 are transcribed in the opposite orientation; their arrangement at the whole-genome level was mapped (Figure 5). All predicted proteins were examined for similarity to known sequences deposited in the public databases of NCBI, and the obtained information was used to provide detailed annotations of the phage proteome. From Figure 5 and Supplementary Table 3, it can be seen that 106 ORFs share apparent database matches with known functions and the rest 158 ORFs are assigned as hypothetical proteins. Aside lysogeny modules, morphogenesis modules (ORFs 9, 10, 43-46, 147-154, 235, 238-240, 242-252, 255-262, and 264) and DNA packaging modules (ORFs 253254) can be clearly identified. However, many ORFs are widely distributed in the phage genome without forming an obvious module, ORFs that are associated with nucleotide metabolism and replication and ORFs that are related to metabolic correlation (Figure 5). The host lysis system of P-KP2 is composed of four parts, $\mathrm{T}$ holin lysis mediator (ORF48), spanin (ORF123 and ORF124), antiholin (ORF180), and endolysin (ORF197), which is consistent with the characteristics of the lysis modules of "KP15likevirus" genus (Zhao et al., 2019).

Most of the gene coding sites of P-KP2 show extremely high homology with their homologous phage, but there are some exceptions. Several loci (including ORFs 3, 20-21, 85, 135-136, and 206 of P-KP2) show different dissimilarity with homologous phages due to the deletion or insertion of a few bases. For example, P-KP2 gp85 (54,624-55,700 bp) has >98\% coverage with homologous phages listed in Table $\mathbf{1}$ at nucleotide level. However, P-KP2 ORF85 shows only 63-72\% coverage with these phages. Actually, "C54409" and "T54410" in P-KP2 were inserted compared with homologous phages. Additionally, lacking one "C" between G11040 and C11041 compared with homologous phages, ORF20 and ORF21 were both predicted as parts of RNA ligase 2 .

As a putative tail fiber protein, P-KP2 ORF46 shows less similarity with homologous phages either at the nucleotide or at the protein level (Supplementary Figure 3). HHPred analysis shows that ORF46 has some identity with tail spike of Enterobacteria phage $\mathrm{Mu}$ (976-1050 residues, PDB ID: 3VTN_A).

\section{P-KP2 Shows Protective Effects Against K. pneumoniae Infection in a Mouse Model}

The MLD of intranasal inoculation of K. pneumoniae W-KP2 was determined as $5.0 \times 10^{8} \mathrm{CFU} /$ mouse. To monitor the therapeutic effect of phage $\mathrm{P}-\mathrm{KP} 2$ on acute pneumonia caused by W-KP2, mice were infected with $2 \times$ MLD $\left(1.0 \times 10^{9} \mathrm{CFU} / \mathrm{mouse}\right)$. At the initial stage of infection (12 h), only a small amount of W-KP2 was colonized. However, without therapeutic intervention, the bacterial load in the lung raised to nearly $10^{10} \mathrm{CFU} / \mathrm{g}$ within 5 days (Figure 6A), and a considerable amount of bacteria entered the peripheral blood $\left(6.7 \times 10^{6} \mathrm{CFU} / \mathrm{mL}\right)$ (Figure 6B). These untreated mice developed severe congestion and hemorrhage in the lung tissues as the disease progressed, most of the alveolar structures collapsed and partially disappeared, accompanied by fibrotic lesions (Figure 7A and Supplementary Figure 6). Besides, W-KP2 infection significantly induced the up-regulation of pro-inflammatory cytokines, such as IL-1 $\beta$, IL-6, TNF$\alpha$, and IFN- $\gamma$ in mice at $72 \mathrm{~h}$ after infection (Figure $7 \mathbf{B}$ ). Accompanied by sustained weight loss and declining health status 
TABLE 1 | Global genome comparison of P-KP2 with homologous phages.

\begin{tabular}{|c|c|c|c|c|c|}
\hline & P-KP2 & phiEap-3 & Matisse & KP27 & KP15 \\
\hline Host strain type & Klebsiella pneumoniae & $\begin{array}{l}\text { Enterobacter } \\
\text { aerogenes }\end{array}$ & Klebsiella pneumoniae & Klebsiella pneumoniae & Klebsiella pneumoniae \\
\hline GenBank number & MT157285.1 & KT321315.1 & KT001918.1 & HQ918180.1 & GU295964.1 \\
\hline $\mathrm{G}+\mathrm{C}$ content & $41.9 \%$ & $42.0 \%$ & $41.8 \%$ & $41.8 \%$ & $41.8 \%$ \\
\hline Genome size (bp) & 172,138 & 175,814 & 176,081 & 174,413 & 174,436 \\
\hline Identity of P-KP2 BLASTN & $100 \%$ & $98 \%$ & $99 \%$ & $99 \%$ & $99 \%$ \\
\hline \multirow[t]{2}{*}{ Query coverage of P-KP2 } & $100 \%$ & $97 \%$ & $97 \%$ & $94 \%$ & $96 \%$ \\
\hline & PMBT1 & KOX10 & Miro & phT4A & KOX8 \\
\hline Host strain type & Klebsiella pneumoniae & Klebsiella oxytoca & Klebsiella pneumoniae & Escherichia coli & $\begin{array}{l}\text { Klebsiella oxytoca; } \\
\text { Klebsiella pneumoniae }\end{array}$ \\
\hline GenBank number & NC_042138.1 & MN101223.1 & KT001919.1 & KX130727.1 & MN101221.1 \\
\hline $\mathrm{G}+\mathrm{C}$ content & $41.9 \%$ & $41.7 \%$ & $41.8 \%$ & $41.7 \%$ & $41.9 \%$ \\
\hline Genome size (bp) & 175,206 & 168,074 & 176,055 & 170,698 & 131,200 \\
\hline Identity of P-KP2 BLASTN & $99 \%$ & $98 \%$ & $97 \%$ & $99 \%$ & $99 \%$ \\
\hline Query coverage of P-KP2 & $98 \%$ & $91 \%$ & $96 \%$ & $90 \%$ & $73 \%$ \\
\hline
\end{tabular}

(Supplementary Figure 4), all the mice of this group died within 5 days.

Phage P-KP2 had a concentration-dependent therapeutic effect on lung infection in mice. Neither $1.0 \times 10^{7} \mathrm{PFU} /$ mouse nor $1.0 \times 10^{8} \mathrm{PFU} / \mathrm{mouse}$ of $\mathrm{P}-\mathrm{KP} 2$ was able to eliminate bacteria from the lungs within 7 days (Supplementary Figure 5A). The phage titer in the lungs of mice treated with low concentration of phage $\left(1.0 \times 10^{7} \mathrm{PFU} /\right.$ mouse $)$ was lower than $10^{4} \mathrm{PFU} / \mathrm{g}$, and phages were completely exhausted within 3 days (Supplementary Figure 5C). Moreover, low concentration of phage $\left(1.0 \times 10^{7} \mathrm{PFU} / \mathrm{mouse}\right)$ cannot stop bacterial proliferation and lung damage, leading to weight loss, poor health status and only 20\% survival rate (Supplementary Figures 4, 5D). When the therapeutic concentration was $1.0 \times 10^{9} \mathrm{PFU} /$ mouse, the bacteria loads in the lungs were effectively reduced (Figure 6A) along with the increase of phage titers (Figure 6C). At the same time, the pneumonia symptoms of mice were significantly alleviated and the survival rate increased to $70 \%$ according to the results of 7 days monitoring, which was comparable to the therapeutic effect of gentamicin (Figure 6D). The lungs of gentamicintreated and phage-treated mice showed mild congestion at $72 \mathrm{~h}$ post infection, but the texture remained tough and shiny. The histopathological changes of the two treatment groups had many similarities, both of which showed local capillary dilatation and a small amount of collapse in local alveolar walls, but most of the alveolar structures still in their normal morphology (Figure 7A and Supplementary Figure 6). In spite of P-KP2 $\left(1.0 \times 10^{9} \mathrm{PFU} / \mathrm{mouse}\right)$ was less able to eliminate bacteria than gentamicin within 3 days of infection, phage treatment was more effective in eliminating residual bacteria from the lungs at the late stage of infection (Figure 6A). Unfortunately, we may not be able to obtain better phage treatment results because higher P-KP2 concentrations cannot be obtained. However, it is heartening that mice treated with both gentamicin and phage showed only transient respiratory symptoms and completely survived (Figure 6D). When P-KP2 was used in combination with gentamicin, the bacteria in the lungs was almost completely eliminated within 6 days (Figure 6A). Moreover, the lung tissue status of the dual treatment group never showed obvious pathological changes (Figure 7A and Supplementary Figure 6). Additionally, at $72 \mathrm{~h}$ after infection, the cytokine levels of the double-treated group were comparable to healthy mice, but both gentamicin-treated and phage-treated mice were slightly higher than that in the dual treatment group (Figure 7B). Therefore, for the lung infection in mice caused by $\mathrm{W}-\mathrm{KP} 2$, the above results not only showed that P-KP2 had a good substitution effect on gentamicin, but also suggested that the combined effect of phage and gentamicin was far superior to that of a single administration.

\section{DISCUSSION}

According to the production of capsular polysaccharides (CPSs), $K$. pneumoniae can be divided into two major types: classic K. pneumoniae (cKP) and hypervirulent K. pneumoniae (hvKP). Unlike hvKP strains, which are prevalent in people with normal immunity, cKP strains are mainly found in populations with weakened immunity or immunodeficiency. Derived from the sputum, non-capsulated W-KP2 is a typical cKP strain. However, it is only sensitive to gentamicin in the drug sensitivity test including carbapenems, so it can be defined as a typical MDR K. pneumoniae. Although the virulence of W-KP2 could not be compared with the hypervirulent strains with capsules (especially $\mathrm{K} 1$ and $\mathrm{K} 2$ serotypes), high-dose (1.0 $\left.\times 10^{9} \mathrm{CFU} / \mathrm{mouse}\right)$ challenge through the respiratory tract still caused mice to die within 5 days. Even after 7 days of infection, residual strains could still be detected in gentamicin-treated mice. Therefore, once such MDR K. pneumoniae causes clinical infection, especially the immunodeficiency population as the target, it is likely that the lag of antibiotic treatment (appropriate antibiotics and doses need to be explored) will lead to delayed healing and even poor prognosis. What's more serious is that while medical workers are still struggling with hvKP and MDR-KP, CR-hvKP 


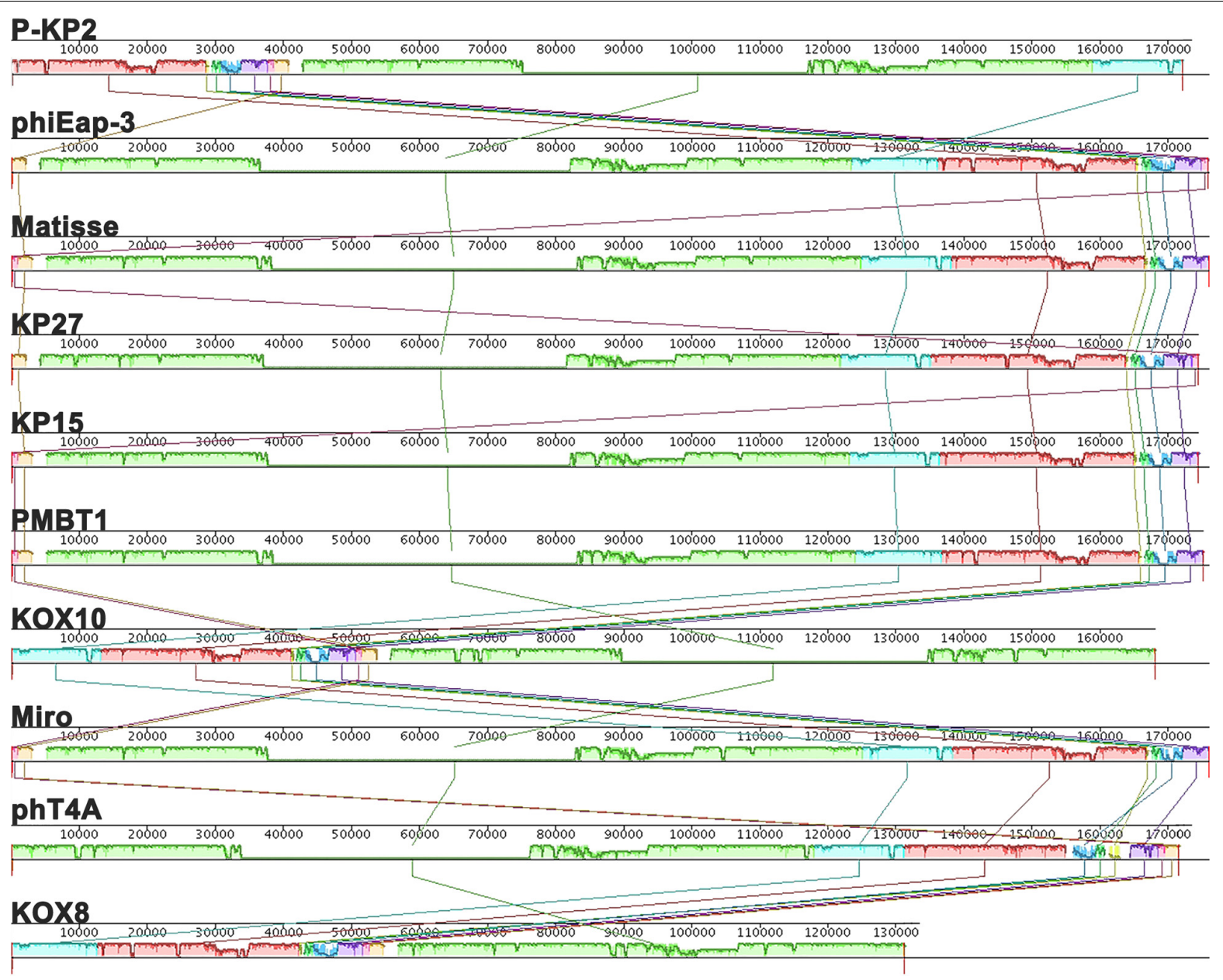

FIGURE 3 | Multiple genome alignments among P-KP2 and homologous phages. The graph was generated by Mauve software. To present the average conservation level in the genome sequence, the similarity of regions is indicated by the height of the bars. Fragment that are not aligned or specific to a particular genome are represented by white areas. Regions of homologous DNA shared by two or more genomes are defined as a local collinear blocks (LCBs), represented by boxes with the identical colors.

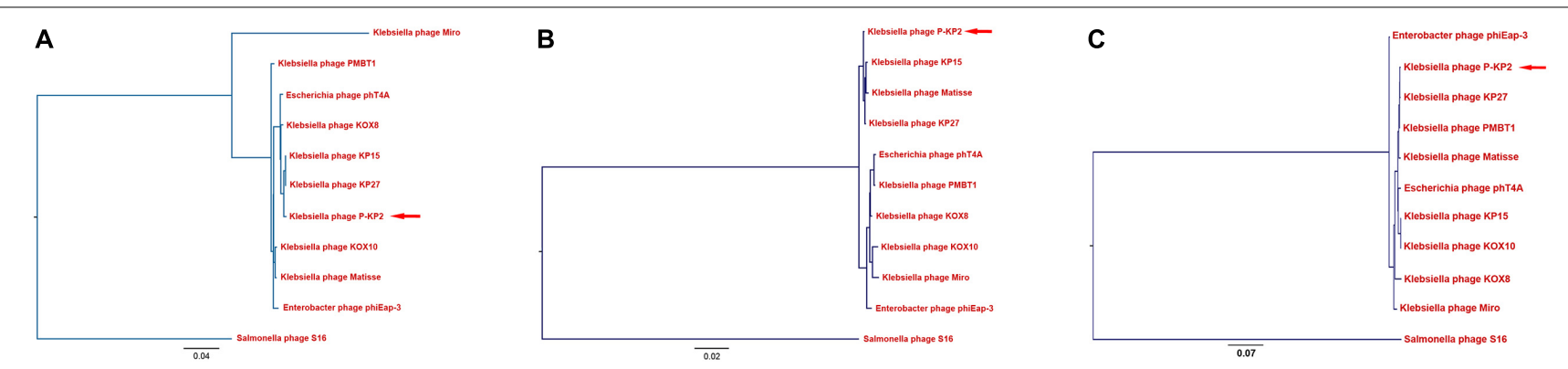

FIGURE 4 | Phylogenetic analyses of P-KP2 and homologous phages. (A) Phylogenetic tree based on terminase large subunit genes. (B) Phylogenetic tree based on genes of major capsid protein. (C) Phylogenetic tree based on genes of DNA polymerase. These sequences were compared using ClustalW, and the phylogenetic tree was generated by PHYLIP version 3.697 (Neighbor-Joining Method, 100 bootstrap replicates). Salmonella phage S16 was selected as the outgroup.

have quietly approached as a new threat, and this new type of strains are largely derived from the horizontal transfer of virulence plasmids or drug-resistant plasmids among strains that have existed for a long time in hospitals or communities. As confirmed by recent studies, some carbapenemase-producing strains, especially members of serotype K47, converted to CRhvKP by obtaining pLVPK-like plasmids (Gu et al., 2018). It can be inferred that W-KP2 also has the possibility to evolve 


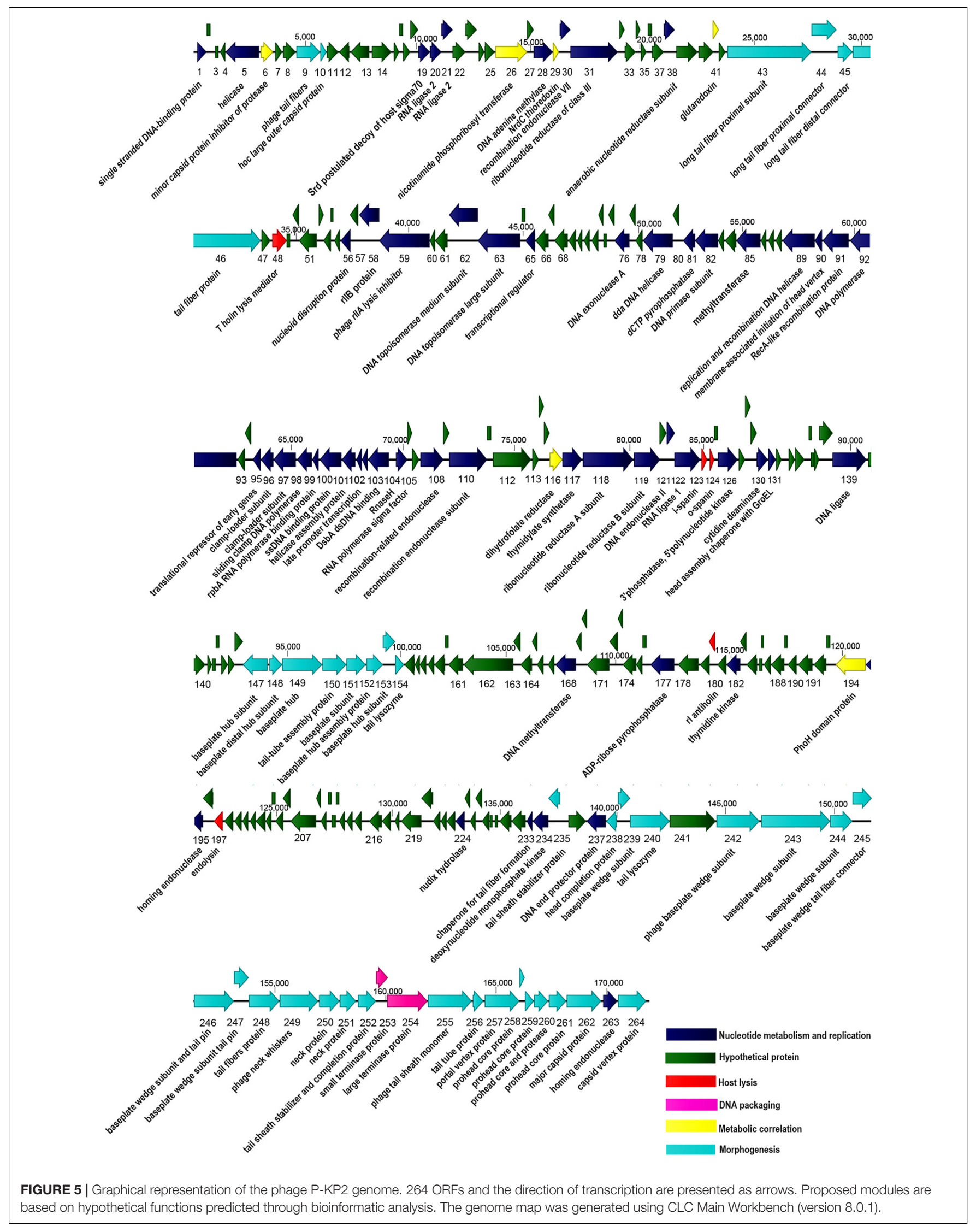




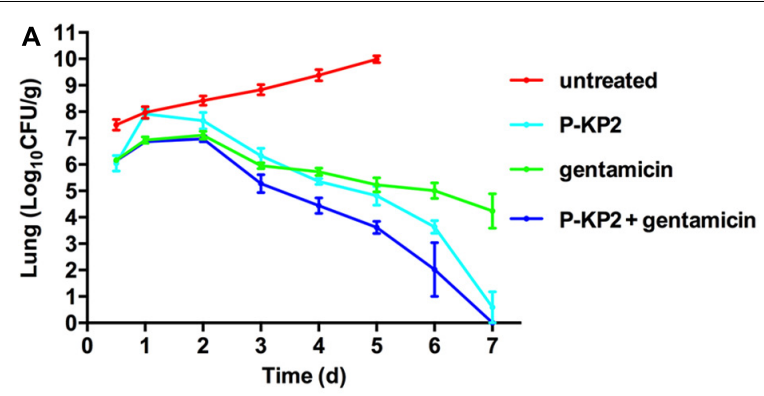

C

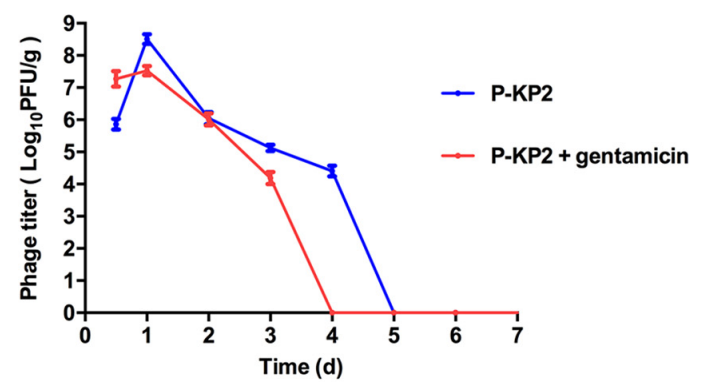

B

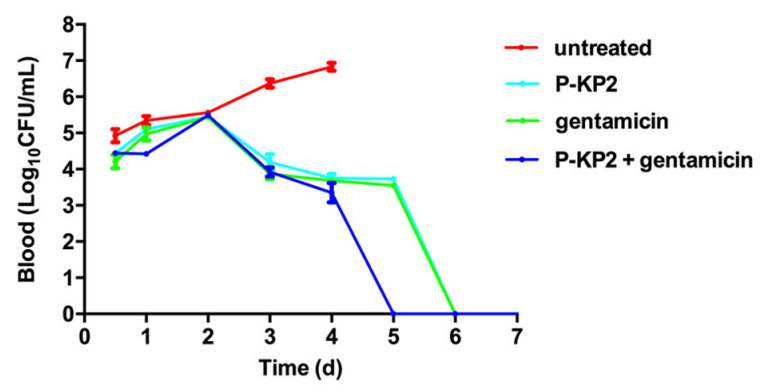

D

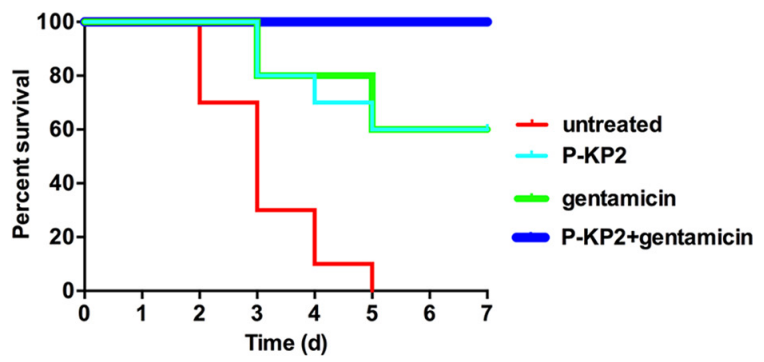

FIGURE 6 | Therapeutic effects of P-KP2 and gentamicin on $\mathrm{K}$. pneumoniae in vivo. All the mice were challenged intranasally with $1.0 \times 10^{9} \mathrm{CFU} / \mathrm{mouse}$ of K. pneumoniae W-KP2. After $1 \mathrm{~h}$ post infection, they were intranasally treated with P-KP2 (1.0 $\left.\times 10^{9} \mathrm{PFU} / \mathrm{mouse}\right)$, gentamicin (1.5 mg/kg), or phage-antibiotic combination (gentamicin was administered intranasally at 30 min after P-KP2 administration), respectively. The untreated group was administered with PBS under the same conditions. (A) Bacterial loads in the lungs. At every $24 \mathrm{~h}$ (lasting for 7 days) after W-KP2 infection, the right lungs of the euthanized mice in each group were carefully separated, weighed and homogenized. Then the bacterial loads in the lung homogenates were detected after serial dilution $(n=3)$. (B) Bacterial loads in blood. Peripheral blood samples $(10 \mu \mathrm{L})$ were obtained from the caudal veins of the anesthetized mice $(n=3)$. (C) Phage titers in the lungs. Phage titers of lung homogenates of each group were detected after serial dilution $(n=3)$. The above data represent the mean \pm SEM of triplicate experiments. (D) Survival rates. Survival rates of W-KP2-infected mice in each group were determined. Each group contained ten mice. Statistical analysis was performed using the Kaplan-Meier method $[P<0.0001$, log-rank (Mantel-Cox) test].

into CR-hvKP. Therefore, this study attempted to control the infection caused by $\mathrm{W}-\mathrm{KP} 2$ in vivo through phage therapy and phage-antibiotic combination therapy and provided a solution to prevent such strains from evolving into CR-hvKP.

P-KP2 has extremely high sequence homology with Enterobacter phage phiEap-3 (Zhao et al., 2019) and Escherichia phage phT4A (Pereira et al., 2017). They all form small plaques with a diameter $\approx 1 \mathrm{~mm}$, but their host spectrums are quite different. The host spectrums of phiEap-3 and phT4A are much wider than that of P-KP2, but they cannot lyse any K. pneumoniae. The host spectrum of phages largely depends on the three-dimensional structure of their receptor-binding proteins (RBPs), especially the C-terminus. With the functions of depolymerase or tail spike protein (TSP), most of RBPs are encoded in the tail fiber protein, and a few are located on the base plate or neck of the phage (Pires et al., 2016). In this study, P-KP2 ORF46, a putative tail fiber protein, had been predicted to be RBP of the phage due to its tail spike-like domain (976-1,050 residues, PDB ID: 3VTN_A), indicating that the protein may mediate specific binding of phage to the host outer membrane proteins ( $\mathrm{Tu}$ et al., 2017). The C-terminus of ORF46 and its homologous proteins have low sequence conservation (Supplementary Figure 3), suggesting that gp46 may be originated from a later evolutionary stage in the process of horizontal gene exchange (Evseev et al., 2020). Besides, the unique C-terminus may determine the huge host spectrum difference between P-KP2 and its homologous phages. In fact, phage P-KP2 can specifically lyse K47 serotype K. pneumoniae. The reason for its narrow host spectrum may be that clinical isolates of this serotype are not common.

The high burst size of P-KP2 (204 PFU/cell) may depend on the multi-gene lysis system composed of $\mathrm{T}$ holin lysis mediator (ORF48), spanin (ORF123 and ORF124), antiholin (ORF180), and endolysin (ORF197). Antiholin can control the timing of host lysis by regulating holin, thereby gaining time for the replication and assembly of more progeny virions, resulting in a larger burst size of phages with multi-gene lysis systems (Cahill and Young, 2019). With such a high burst size and no harmful genes in the genome, P-KP2 has the potential to become a therapeutic agent for K47 serotype K. pneumoniae infection. In our study, P-KP2 treatment $\left(1.0 \times 10^{9} \mathrm{PFU} /\right.$ mouse $)$ alone significantly reduced inflammatory responses and pathological changes in mice with acute pneumonia in vivo. In addition, the survival rate of the group was comparable to that of treated with gentamicin, but it was significantly better in eliminating residual bacteria from lung tissue. Some recent studies have shown that the combined administration of phages and antibiotics is more effective than monotherapy in treating bacterial infections (Segall et al., 2019). Based on this, we attempted to treat acute pneumonia caused by $\mathrm{W}-\mathrm{KP} 2$ with a combination of P-KP2 

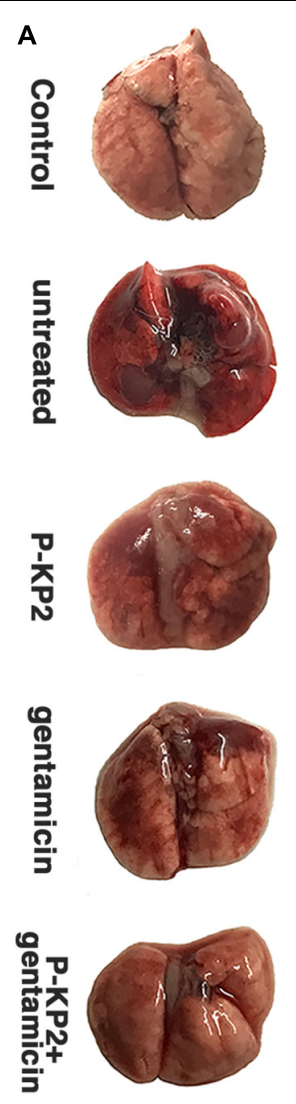
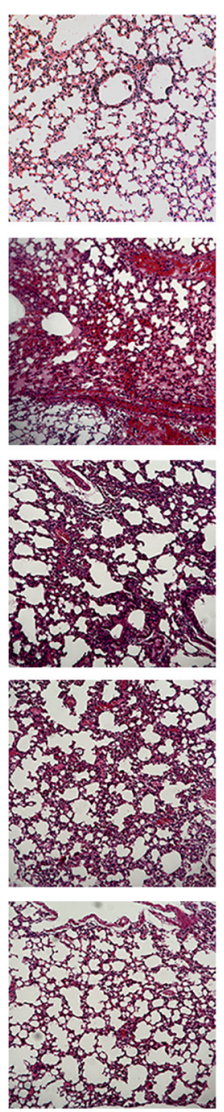

B
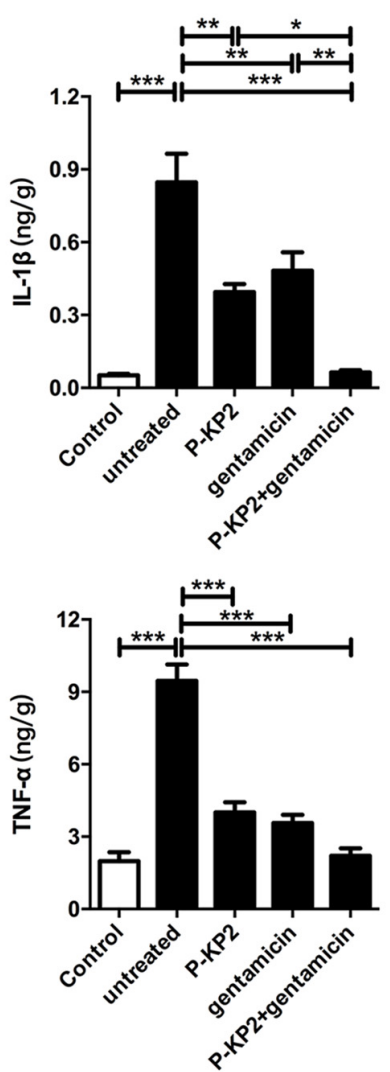

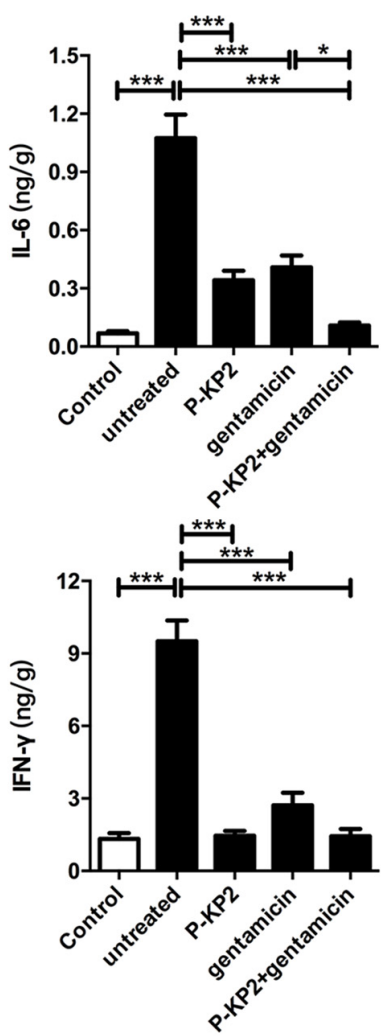

FIGURE 7 | Pathological changes and cytokine levels of mice in different groups. All the mice were challenged intranasally with $1.0 \times 10^{9}$ CFU/mouse of K. pneumoniae W-KP2. After $1 \mathrm{~h}$ post infection, they were intranasally treated with P-KP2 (1.0 × $\left.10^{9} \mathrm{PFU} / \mathrm{mouse}\right)$, gentamicin (1.5 mg/kg), or phage-antibiotic combination (gentamicin was administered intranasally at 30 min after P-KP2 administration), respectively. The untreated group was administered with PBS under the same conditions. (A) Pathological observation. At $72 \mathrm{~h}$ after W-KP2 infection, the lungs of the euthanized mice in each group were photographed after careful removal, and the sections of left lung tissues were stained with H\&E (magnification, $\times 100)$. Lung tissue of healthy mice was served as a control. (B) Determination of cytokine levels. At $72 \mathrm{~h}$ after W-KP2 infection, cytokine levels (IL-1 $\beta$, IL-6, TNF- $\alpha$, and IFN- $\gamma$ ) in lung homogenates of mice treated with phage P-KP2, gentamicin, or phage-antibiotic combination were determined. Lung tissue homogenates of healthy mice were served as controls. * , **, and ${ }^{* \star *}$ represent significant differences at $P<0.05, P<0.01$, and $P<0.001$, respectively. Data represent the mean \pm SEM of triplicate experiments.

and gentamicin. Encouragingly, not only did all the mice of the combined therapy group survive, but the pathological changes and inflammatory responses in the lungs were maintained at normal levels, possibly due to the effective inhibition of bacterial proliferation and complete elimination of residual bacteria by phage-antibiotic combination therapy. As an important reason for limiting the therapeutic effect of P-KP2 alone, this phage was easily eliminated by the immune system (Supplementary Figure 5C), and gentamicin seems to play a leading role in our combination therapy. However, phages specifically alter the surface structures of bacteria (polysaccharides, outer membrane proteins, etc.), thereby clearing the barriers for the infiltration of some antibiotics, indicating that phages have the effect of enhancing the sensitivity of bacteria to antibiotics (Segall et al., 2019). Therefore, the synergistic effect of phages on antibiotics is the key to the outstanding therapeutic effect of this combination therapy.

From the main way of treating bacterial infections worldwide, it will take time for us to get rid of dependence on antibiotics completely. However, both the combination of high-dose antibiotics and the treatment with new antibiotics may increase suffering and treatment costs for patients. Therefore, in the context of "weak" antibiotic efficacy, it is more realistic to seek ways to enhance the therapeutic effect of "low-grade" antibiotics. In summary, our study provided a clear case for phage-antibiotic combination against MDR K. pneumoniae infections and pointed out the direction to curb the emergence of more CR-hvKP, which has important theoretical significance and practical value.

\section{CONCLUSION}

The threat of MDR $K$. pneumoniae to public health in humans, especially in immunocompromised populations, and its evolution toward CR-hvKP have led to increased public recognition in the substitution or synergism of phage therapy for antibiotics. In this study, MDR $K$. pneumoniae W-KP2 was used as the host to isolate a novel myophage P-KP2 belonging to "KP15 virus" family, and then its biological characteristics 
and genomics information were analyzed. Due to its high burst size and the absence of harmful genes, P-KP2 was analyzed as a candidate therapeutic agent against acute pneumonia caused by $\mathrm{W}-\mathrm{KP} 2$. When administered with $1.0 \times 10^{9} \mathrm{PFU} / \mathrm{mouse}$, the phage proliferated rapidly and completely eliminated the bacteria in the lungs within 7 days, which exceeded the therapeutic effect of gentamicin. More encouragingly, the combination of P-KP2 and gentamicin not only rescued all infected mice, but also effectively inhibited the development of inflammation. Therefore, as the first case of "KP15 virus" family phage to be applied for treatment, the processes of $\mathrm{P}-\mathrm{KP} 2$ proliferation, promotion of bacterial elimination have been elucidated in vivo, which not only filled the gap in the phage library against K47 serotype K. pneumoniae infection, but also provided a theoretical basis for the subsequent phage-antibiotic combination therapies and blocking the occurrence of more CR-hvKP.

\section{DATA AVAILABILITY STATEMENT}

The completed genome sequence of Klebsiella phage P-KP2 has been deposited in the GenBank database under accession number MT157285.

\section{ETHICS STATEMENT}

The animal study was reviewed and approved by the Care and Use of Laboratory Animals of the Jilin University.

\section{REFERENCES}

Abedon, S. T. (2019). Phage-antibiotic combination treatments: antagonistic impacts of antibiotics on the pharmacodynamics of phage therapy? Antibiotics (Basel) 8:182. doi: 10.3390/antibiotics 8040182

Altschul, S. F., Madden, T. L., Schaffer, A. A., Zhang, J., Zhang, Z., Miller, W., et al. (1997). Gapped BLAST and PSI-BLAST: a new generation of protein database search programs. Nucleic Acids Res. 25, 3389-3402. doi: 10.1093/nar/25.17.3389

Anand, T., Virmani, N., Kumar, S., Mohanty, A. K., Pavulraj, S., Bera, B. C., et al. (2020). Phage therapy for treatment of virulent Klebsiella pneumoniae infection in a mouse model. J. Glob. Antimicrob. Resist. 21, 34-41. doi: 10.1016/j.jgar. 2019.09.018

Bao, J., Wu, N., Zeng, Y., Chen, L., Li, L., Yang, L., et al. (2020). Non-active antibiotic and bacteriophage synergism to successfully treat recurrent urinary tract infection caused by extensively drug-resistant Klebsiella pneumoniae. Emerg. Microbes Infect. 9, 771-774. doi: 10.1080/22221751.2020.1747950

Besemer, J., Lomsadze, A., and Borodovsky, M. (2001). GeneMarkS: a self-training method for prediction of gene starts in microbial genomes. Implications for finding sequence motifs in regulatory regions. Nucleic Acids Res. 29, 2607-2618. doi: 10.1093/nar/29.12.2607

Cahill, J., and Young, R. (2019). Phage lysis: multiple genes for multiple barriers. Adv. Virus Res. 103, 33-70. doi: 10.1016/bs.aivir.2018.09.003

Cai, R., Wang, G., Le, S., Wu, M., Cheng, M., Guo, Z., et al. (2019a). three capsular polysaccharide synthesis-related glucosyltransferases, gt-1, gt-2 and wcaj, are associated with virulence and phage sensitivity of Klebsiella pneumoniae. Front. Microbiol. 10:1189. doi: 10.3389/fmicb.2019.01189

Cai, R., Wang, Z., Wang, G., Zhang, H., Cheng, M., Guo, Z., et al. (2019b). Biological properties and genomics analysis of vB_KpnS_GH-K3, a Klebsiella phage with a putative depolymerase-like protein. Virus Genes 55, 696-706. doi: 10.1007/s11262-019-01681-z

\section{AUTHOR CONTRIBUTIONS}

GW, ZG, YJ, XL, YG, HZ, HX, RZ, LB, SL, and LY assisted in carrying out the experiment. $\mathrm{ZW}$ and $\mathrm{RC}$ wrote the manuscript. $\mathrm{XF}, \mathrm{CS}, \mathrm{LL}, \mathrm{WH}$, and JG helped with the design of experimental ideas and the revision of manuscripts. All authors contributed to the article and approved the submitted version.

\section{FUNDING}

This work was financially supported through grants from the National Natural Science Foundation of China (Grant Nos. 32072824, 31872505, and U19A2038), the Natural Science Foundation of Jilin Province (Changchun, China; Grant No. 20200201120JC), the Jilin Province Science Foundation for Youths (Changchun, China; Grant No. 20190103106JH), the Achievement Transformation Project of the First Hospital of Jilin University (No. JDYYZH-1902025), the Shanghai Municipal Health Commission Scientific Research Project (Grant No. 20194Y0061), and the Fundamental Research Funds for the Central Universities.

\section{SUPPLEMENTARY MATERIAL}

The Supplementary Material for this article can be found online at: https://www.frontiersin.org/articles/10.3389/fmicb. 2021.674068/full\#supplementary-material

Chadha, P., Katare, O. P., and Chhibber, S. (2017). Liposome loaded phage cocktail: enhanced therapeutic potential in resolving Klebsiella pneumoniae mediated burn wound infections. Burns 43, 1532-1543. doi: 10.1016/j.burns.2017.03.029

Cheng, M., Liang, J., Zhang, Y., Hu, L., Gong, P., Cai, R., et al. (2017). The Bacteriophage EF-P29 efficiently protects against lethal vancomycin-resistant enterococcus faecalis and alleviates gut microbiota imbalance in a murine bacteremia model. Front. Microbiol. 8:837. doi: 10.3389/fmicb.2017.00837

Darling, A. C., Mau, B., Blattner, F. R., and Perna, N. T. (2004). Mauve: multiple alignment of conserved genomic sequence with rearrangements. Genome Res. 14, 1394-1403. doi: 10.1101/gr.2289704

Davies, E. V., Winstanley, C., Fothergill, J. L., and James, C. E. (2016). The role of temperate bacteriophages in bacterial infection. FEMS Microbiol. Lett. 363:fnw015. doi: 10.1093/femsle/fnw015

Evseev, P. V., Lukianova, A. A., Shneider, M. M., Korzhenkov, A. A., Bugaeva, E. N., Kabanova, A. P., et al. (2020). Origin and evolution of studiervirinae bacteriophages infecting pectobacterium: horizontal transfer assists adaptation to new niches. Microorganisms 8:1707. doi: 10.3390/microorganisms8111707

Feldman, A. T., and Wolfe, D. (2014). Tissue processing and hematoxylin and eosin staining. Methods Mol. Biol. 1180, 31-43. doi: 10.1007/978-1-4939-1050-2_3

Gadagkar, R., and Gopinathan, K. P. (1980). Bacteriophage burst size during multiple infections. J. Biosci. 2, 253-259. doi: 10.1007/BF02703251

Garneau, J. R., Depardieu, F., Fortier, L. C., Bikard, D., and Monot, M. (2017). PhageTerm: a tool for fast and accurate determination of phage termini and packaging mechanism using next-generation sequencing data. Sci. Rep. 7:8292. doi: 10.1038/s41598-017-07910-5

Gong, P., Cheng, M., Li, X., Jiang, H., Yu, C., Kahaer, N., et al. (2016). Characterization of Enterococcus faecium bacteriophage IME-EFm5 and its endolysin LysEFm5. Virology 492, 11-20. doi: 10.1016/j.virol.2016.02.006

Gu, D., Dong, N., Zheng, Z., Lin, D., Huang, M., Wang, L., et al. (2018). A fatal outbreak of ST11 carbapenem-resistant hypervirulent Klebsiella pneumoniae in 
a Chinese hospital: a molecular epidemiological study. Lancet Infect. Dis. 18, 37-46. doi: 10.1016/S1473-3099(17)30489-9

Hampton, H. G., Watson, B. N. J., and Fineran, P. C. (2020). The arms race between bacteria and their phage foes. Nature 577, 327-336. doi: 10.1038/s41586-0191894-8

Hodyra-Stefaniak, K., Miernikiewicz, P., Drapala, J., Drab, M., Jonczyk-Matysiak, E., Lecion, D., et al. (2015). Mammalian host-versus-phage immune response determines phage fate in vivo. Sci. Rep. 5:14802. doi: 10.1038/sre14802

Hsu, C. R., Lin, T. L., Pan, Y. J., Hsieh, P. F., and Wang, J. T. (2013). Isolation of a bacteriophage specific for a new capsular type of Klebsiella pneumoniae and characterization of its polysaccharide depolymerase. PLoS One 8:e70092. doi: 10.1371/journal.pone.0070092

Ji, Y., Cheng, M., Zhai, S., Xi, H., Cai, R., Wang, Z., et al. (2019). Preventive effect of the phage VB-SavM-JYL01 on rabbit necrotizing pneumonia caused by Staphylococcus aureus. Vet. Microbiol. 229, 72-80. doi: 10.1016/j.vetmic.2018. 12.021

Kaabi, S. A. G., and Musafer, H. K. (2019). An experimental mouse model for phage therapy of bacterial pathogens causing bacteremia. Microb. Pathog. 137:103770. doi: 10.1016/j.micpath.2019.103770

Koberg, S., Brinks, E., Fiedler, G., Husing, C., Cho, G. S., Hoeppner, M. P., et al. (2017). Genome sequence of Klebsiella pneumoniae bacteriophage PMBT1 isolated from raw sewage. Genome Announc. 5:e00914-16. doi: 10.1128/ genomeA.00914-16

Lin, T. L., Hsieh, P. F., Huang, Y. T., Lee, W. C., Tsai, Y. T., Su, P. A., et al. (2014). Isolation of a bacteriophage and its depolymerase specific for K1 capsule of Klebsiella pneumoniae: implication in typing and treatment. J. Infect. Dis. 210, 1734-1744. doi: 10.1093/infdis/jiu332

Margulies, M., Egholm, M., Altman, W. E., Attiya, S., Bader, J. S., Bemben, L. A., et al. (2005). Genome sequencing in microfabricated high-density picolitre reactors. Nature 437, 376-380. doi: 10.1038/nature03959

Mijalis, E. M., Lessor, L. E., Cahill, J. L., Rasche, E. S., and Kuty Everett, G. F. (2015). Complete genome sequence of Klebsiella pneumoniae carbapenemaseproducing K. pneumoniae myophage miro. Genome Announc. 3:e01137-15. doi: 10.1128/genomeA.01137-15

Navon-Venezia, S., Kondratyeva, K., and Carattoli, A. (2017). Klebsiella pneumoniae: a major worldwide source and shuttle for antibiotic resistance. FEMS Microbiol. Rev. 41, 252-275. doi: 10.1093/femsre/fux013

Nobrega, F. L., Costa, A. R., Kluskens, L. D., and Azeredo, J. (2015). Revisiting phage therapy: new applications for old resources. Trends Microbiol. 23, 185191. doi: 10.1016/j.tim.2015.01.006

Paczosa, M. K., and Mecsas, J. (2016). Klebsiella pneumoniae: going on the offense with a strong defense. Microbiol. Mol. Biol. Rev. 80, 629-661. doi: 10.1128/ mmbr.00078-15

Pan, Y. J., Lin, T. L., Chen, C. T., Chen, Y. Y., Hsieh, P. F., Hsu, C. R., et al. (2015). Genetic analysis of capsular polysaccharide synthesis gene clusters in 79 capsular types of Klebsiella spp. Sci. Rep. 5:15573. doi: 10.1038/srep 15573

Pan, Y. J., Lin, T. L., Chen, Y. H., Hsu, C. R., Hsieh, P. F., Wu, M. C., et al. (2013). Capsular types of Klebsiella pneumoniae revisited by wzc sequencing. PLoS One 8:e80670. doi: 10.1371/journal.pone.0080670

Pereira, C., Moreirinha, C., Lewicka, M., Almeida, P., Clemente, C., Romalde, J. L., et al. (2017). Characterization and in vitro evaluation of new bacteriophages for the biocontrol of Escherichia coli. Virus Res. 227, 171-182. doi: 10.1016/j. virusres.2016.09.019
Pires, D. P., Oliveira, H., Melo, L. D., Sillankorva, S., and Azeredo, J. (2016). Bacteriophage-encoded depolymerases: their diversity and biotechnological applications. Appl. Microbiol. Biotechnol. 100, 2141-2151. doi: 10.1007/s00253015-7247-0

Provasek, V. E., Lessor, L. E., Cahill, J. L., Rasche, E. S., and Kuty Everett, G. F. (2015). Complete genome sequence of carbapenemase-producing Klebsiella pneumoniae myophage matisse. Genome Announc. 3:e01136-15. doi: 10.1128/ genomeA.01136-15

Sambrook, J., and Russell, D. W. (2006). Precipitation of Bacteriophage Lambda Particles from large-scale lysates. CSH Protoc. 2006:pdb.rot3966. doi: 10.1101/ pdb.prot3966

Segall, A. M., Roach, D. R., and Strathdee, S. A. (2019). Stronger together? Perspectives on phage-antibiotic synergy in clinical applications of phage therapy. Curr. Opin. Microbiol. 51, 46-50. doi: 10.1016/j.mib.2019.0 3.005

Shimada, M. K., and Nishida, T. (2017). A modification of the PHYLIP program: a solution for the redundant cluster problem, and an implementation of an automatic bootstrapping on trees inferred from original data. Mol. Phylogenet. Evol. 109, 409-414. doi: 10.1016/j.ympev.2017.02.012

Stothard, P., and Wishart, D. S. (2005). Circular genome visualization and exploration using CGView. Bioinformatics 21, 537-539. doi: 10.1093/ bioinformatics/bti054

Sunagawa, S., DeSantis, T. Z., Piceno, Y. M., Brodie, E. L., DeSalvo, M. K., Voolstra, C. R., et al. (2009). Bacterial diversity and White Plague Disease-associated community changes in the Caribbean coral Montastraea faveolata. ISME J. 3, 512-521. doi: 10.1038/ismej.2008.131

Tu, J., Park, T., Morado, D. R., Hughes, K. T., Molineux, I. J., and Liu, J. (2017). Dual host specificity of phage SP6 is facilitated by tailspike rotation. Virology 507, 206-215. doi: 10.1016/j.virol.2017.04.017

Xia, F., Li, X., Wang, B., Gong, P., Xiao, F., Yang, M., et al. (2016). Combination therapy of LysGH15 and apigenin as a new strategy for treating pneumonia caused by staphylococcus aureus. Appl. Environ. Microbiol. 82, 87-94. doi: 10.1128/AEM.02581-15

Zhao, J., Zhang, Z., Tian, C., Chen, X., Hu, L., Wei, X., et al. (2019). Characterizing the biology of lytic bacteriophage vb_eaem_phieap-3 infecting multidrugresistant Enterobacter aerogenes. Front. Microbiol. 10:420. doi: 10.3389/fmicb. 2019.00420

Zimmermann, L., Stephens, A., Nam, S. Z., Rau, D., Kubler, J., Lozajic, M., et al. (2018). A completely reimplemented MPI bioinformatics toolkit with a new HHpred server at its core. J. Mol. Biol. 430, 2237-2243. doi: 10.1016/j.jmb.2017. 12.007

Conflict of Interest: The authors declare that the research was conducted in the absence of any commercial or financial relationships that could be construed as a potential conflict of interest.

Copyright (c) 2021 Wang, Cai, Wang, Guo, Liu, Guan, Ji, Zhang, Xi, Zhao, Bi, Liu, Yang, Feng, Sun, Lei, Han and Gu. This is an open-access article distributed under the terms of the Creative Commons Attribution License (CC BY). The use, distribution or reproduction in other forums is permitted, provided the original author(s) and the copyright owner(s) are credited and that the original publication in this journal is cited, in accordance with accepted academic practice. No use, distribution or reproduction is permitted which does not comply with these terms. 